\title{
A Study on the Sow Breeding Management System for MSY Improvement based QR-Code
}

\author{
Hansol Park ${ }^{1}$, Hoseok Jeong ${ }^{2}$ and Hyun Yoe ${ }^{3 *}$ \\ ${ }^{123}$ Department of Information and Communication Engineering, \\ Sunchon National University, Korea \\ \{hansol6618,hsjeong,yhyun\}@sunchon.ac.kr
}

\begin{abstract}
Domestic hog raising industry has been continuously advancing both quantitatively and qualitatively along with economic advancement. However, the current circumstance is that system on domestic sow specification management is insufficient with severe individual differences, and the number of MSY remains to be low compared to that of advanced countries. To solve this issue, systematic specification management technology is needed to decrease the replacement rate of sow and improve the parturition and productivity. Through the proposed QR code based sow specification management system in this paper, it increased the weekly management cycle of sow and designed a system for systematic specification management of sow. When pregnands succeeded through sow weekly management, 16 weeks of pregnancy period stored in database and current date are added to provide expected delivery date to user. When pregnancy failed, it is added with rut cycle 22 days to display expected date of rut. Through this, it con increase the number of MSY and provide convenience of user.
\end{abstract}

Keywords: Sow Breeding Managenent, Application, System, PC

\section{Introduction}

This Domestic hog raising industry has been advancing continuously not only quantitatively along with economic advancement but also qualitatively through high-quality safe pork production. However, competition against hog raising advancement countries is inevitable due to opening of market from FTA (Free Trade Agreement) and domestic hog raising industry is experiencing difficulties due to increase in internal grain price [1-3].

In the comparison of production amount with hog raising advancement countries, MSY of Holland is 26.5 and that of Denmark is 25, whereas that of Korea is 15.5 with low production amount. The reason for the low sow productivity of our country is due to lack of systematic specification management technology. In addition, there are many difficulties in managing specification in real-time or obtaining accurate data because the majority of domestic hog raising farms are doing sow weekly management and specification management manually [4$6]$.

To solve this issue, systematic specification management technology is needed to decrease the replacement rate of sow and improve the parturition and productivity. Accordingly, this paper will propose QR code based sow specification management system for MSY improvement to promote the enhancement of sow parturition and productivity.

${ }^{*}$ Corresponding author 
The QR code of proposed system is able to store high volume of information and print in small space with excellent restoration capability and continuous function support. In addition, it can restore damages in the range of each level of about 7 30\% because of its superior error restoration capability [7].

In the case where the QR code attached to sow room has been lost, its restoration is possible and it can solve the difficulties of hog raising farm on the issue of using expensive RFID tag. In addition, sow specification management is possible anytime, anywhere through mobile device.

The proposed system extracts the key value stored upon recognizing the QR code installed in each sow room and stores the registration information of sow by using the extracted key value. The registration information of sow consists of expected rut date, daily feed intake amount, weight, back fat thickness and vaccine status and it is stored in the database of integrated management server. On the expected rut date of sow, it provides hotification service to user to allow artificial insemination at optimal period. In addition, it provides expected delivery date when pregnancy succeeded upon entering the pregnancy status of sow by user, and it provides the next rut cycle whem pregnancy failed User can conduct systematic specification management through $\mathrm{PC}$ and smart device as well as provides convenience to user.

The composition of this paper is as follows Chapter 2 explains the proposed system composition diagram, process, class diagram and database table, and Chapter 3 explains the implementation result of systematic soycspocification management system through PC and mobile device. Lastly, Chapter 4 will conclude the paper with conclusion.

\section{System Design}

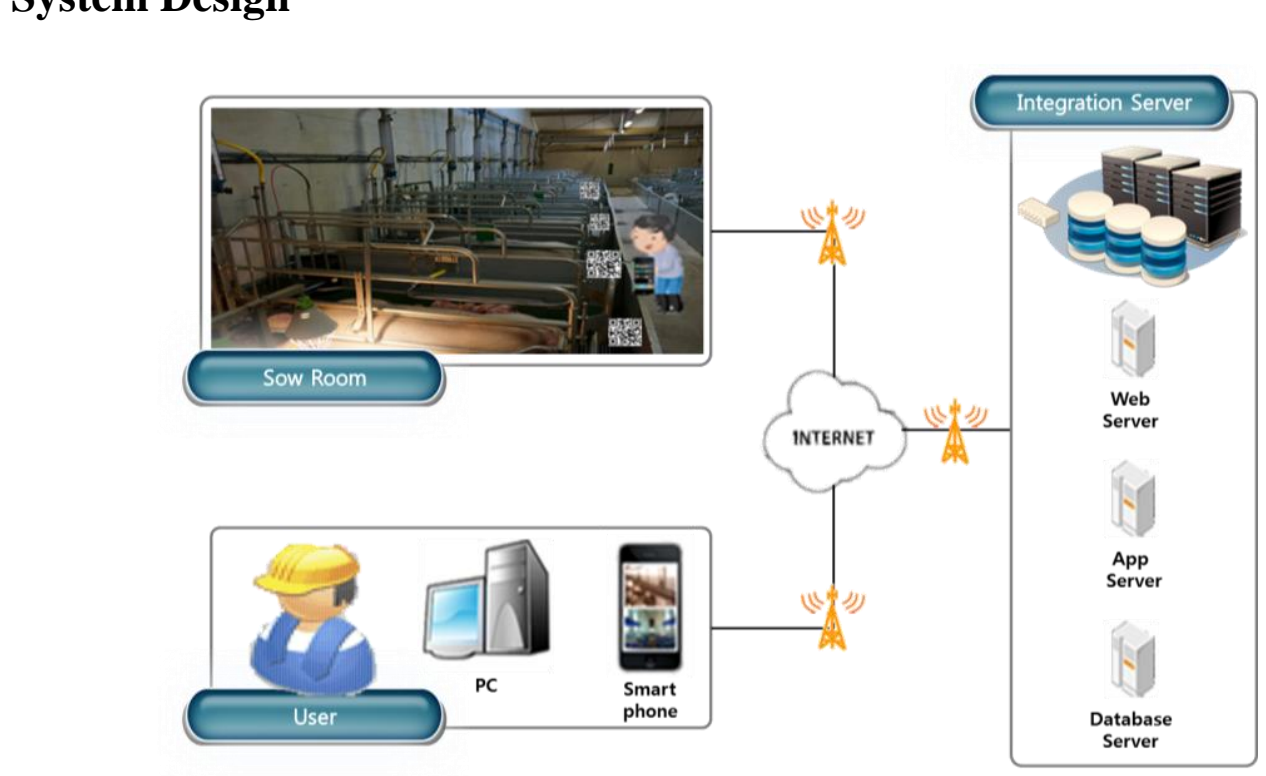

Figure 1. Diagram of the proposed sow breeding management system

Figure 1 is a composition diagram of the proposed sow specification management system. The sow specification management system consists of sow room, integrated management server and user. QR code is attached to each sow room and stored key value is extracted upon recognizing QR code by user to store input information in integrated management server such as the expected rut date of sow, daily feed intake 
amount and weight. The integrated management server used TCP/IP socket communication to allow integration with PC, smart phone and database, and transmitted sow information is processed according to format to store it in database. Through the middleware of PC, users can management the sow specification within stall and conveniently register and management sow information at anywhere through smart phone.

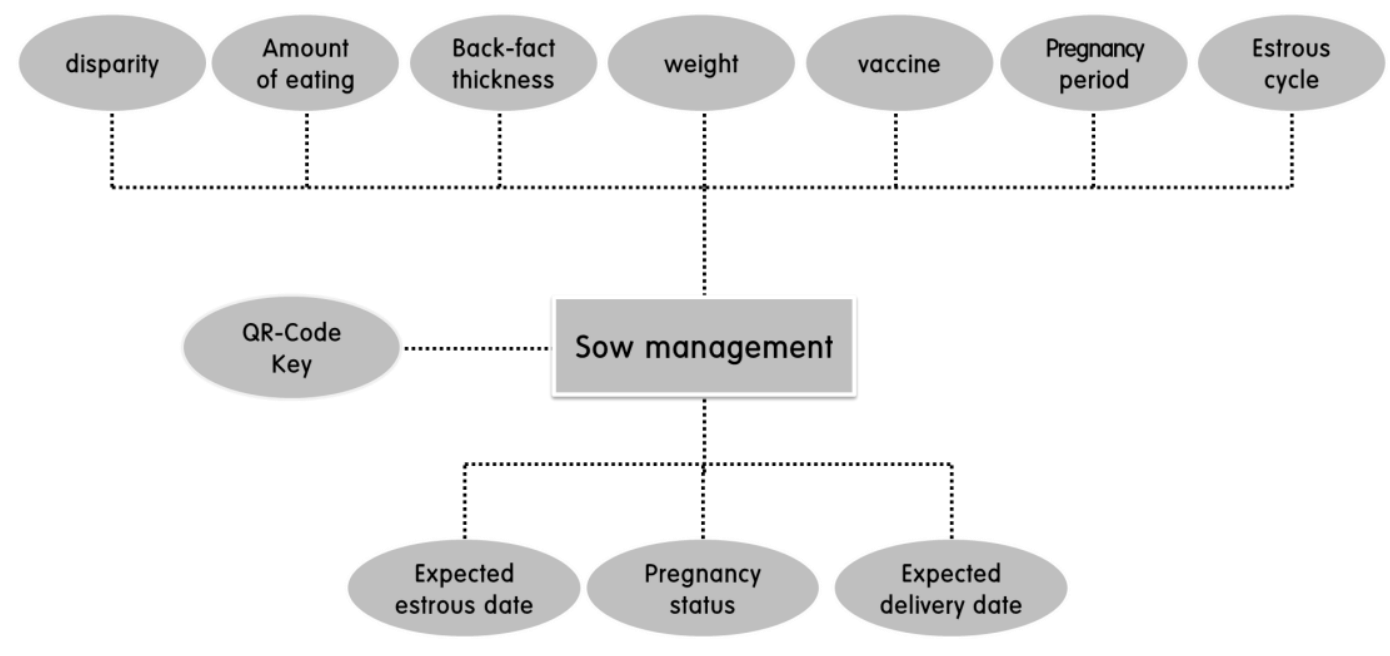

Figure 2. Diagram of the database table structure

Figure 2 is the database table structure diagram of integrated management server. The composition of table Consists of QR code extracted key value, daily feed intake amount, vaccine status, weight, back fat thickness, parturition, expected date of rut, pregnancy status, expected delixery date, rut cycle and pregnancy period. Since the number of weaning pig is determined according to sow's daily feed intake amount, back fat thickness and yeight, it requires the management of user. It consists of rut cycle and pregnancy period table to automatically calculate the expected delivery date and expected rut date according to pregnancy status.

Figure 3 is the process of sow specification management system. The user ID and password entered initially during login activity are transmitted to the JSP of web server within integrated management server. It conducts validity test to check the consistency between the-password of data found upon searching data that corresponds with the user ID transmitted to JSP and entered password. After the validity test ends, success or failure result is returned to user GUI at integrated management server and it moves to sow specification management menu screen when succeeded and moves to login screen once again when failed.

Using smart phone, user extracts the stored key value upon recognizing the QR code of sow room and it's in the database of integrated management server. Using the extracted key value, user transmits input information such as the expected rut date of sow, weight and parturition to integrated management server that processes and stores the input information according to format. In addition, it provides notification service to allow artificial insemination at optimal period on the expected rut date of sow. Using PC and smart phone, user can systematically manage specification and improve the productivity of sow. 


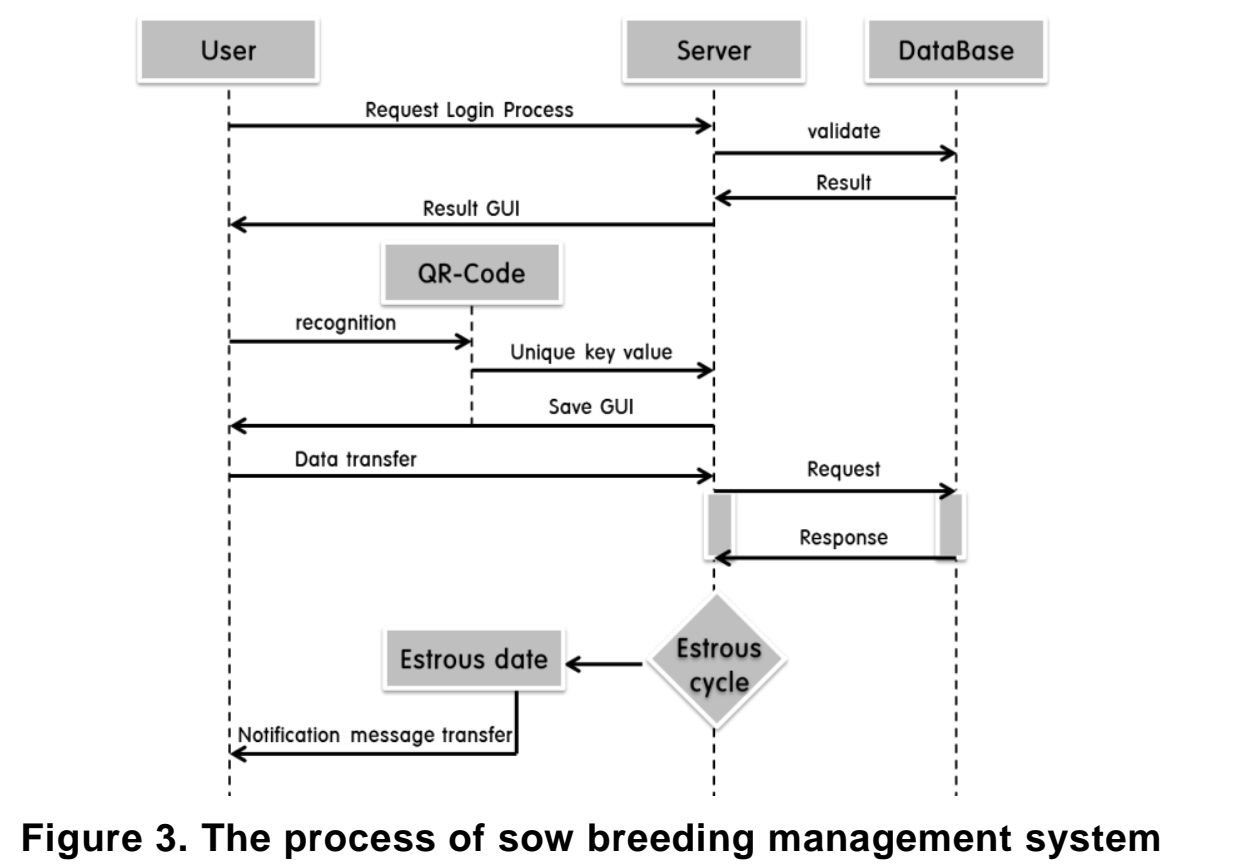

Figure 4 is a class diagram showing the relation hip between classes by showing the method used of class implemented in this system. Initial user uses TCP/IP communication to connect with server. After a successful login, QR code key class is used to recognize QR code to extract the key value stored in $\mathrm{QR}$ code to transmit it to Controller class. In Controller class, key value extracted from QR code and input information on soware transmitte to Model class in get, post method. In Model class, it is integrated with the database of server to store and return the value entered by user for output to user.

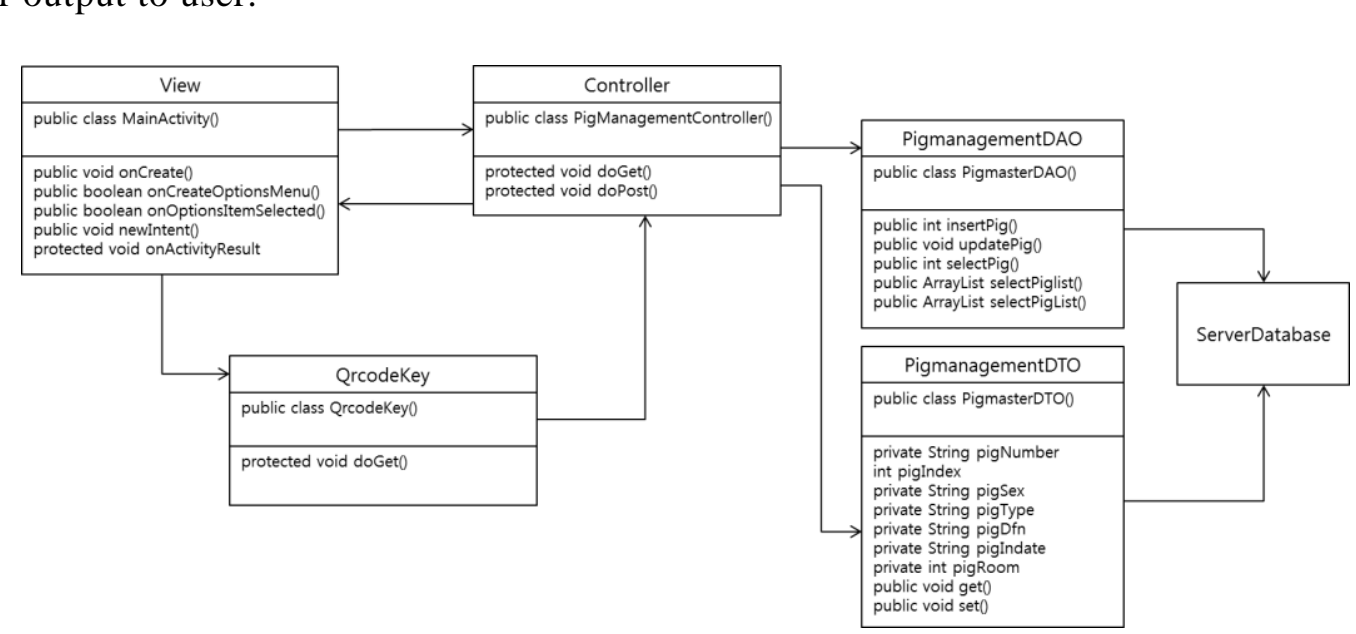

Figure 4. Class Diagram of sow breeding management system

\section{Implementation}

As for the program to implement the middleware of the sow specification management system proposed in this paper, Visual C\# 2010 Express was used. In 
addition, Eclipse Indigo version was used as a program for developing smart phone application, and Apache-tomcat-7.0.42 version and Android 4.0.4 Ice-cream Sandwich version were used as development environment. To recognize QR code, Open API was used, and MySQL Database 5.6 version was used to store sow information in the database of integrated management server.

Figure 5 is screen of the middle implementation of the sow specification management system proposed. Upon recognizing QR code through smart phone, entered information such as sow's daily feed intake amount, vaccine status, weight, back fat thickness, parturition, expected rut date and pregnancy status can be monitored at the userf's PC: In addition, it automatically calculates the expected delivery date and expected rut date according to successful pregnancy of sow for convenient management by user.

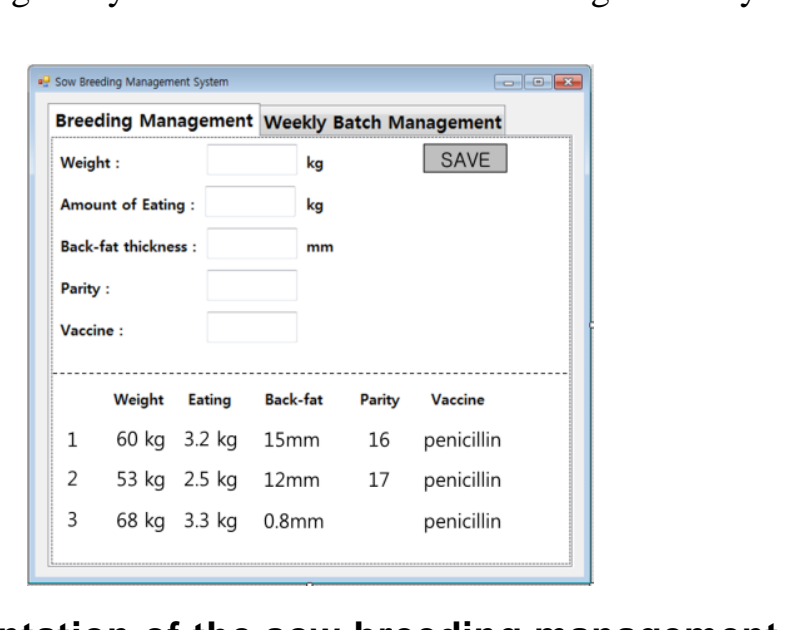

Figure 5. Implementation of the sow breeding management PC client

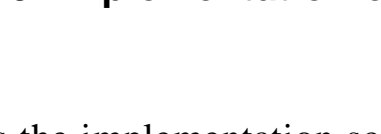
system

Figure 6 is the implementation screen of the smart phone application of the proposed sow specification management system. Using left figure, sow's specification information can be managed and inquired through sow weight, daily feed intake amount, back fat thickness, vaccine status and parturition value for the specification management of sow. Using right figure for the weekly management of sow, it has been implemented to allow artificial insemination during optimal period. Since rut cycle of sow is 19 23 days and rut continuation time is only about 40 60 hours, there are many case of missing the optimal rut period. In the case where current date and the expected rut date of sow coincide, it transmits notification message to user to allow artificial insemination during optimal period. When sow pregnancy succeeded, the pregnancy period of 16 weeks (112 days) stored in the database and current date are added together to provide expected delivery date to user. When pregnancy failed, it displays expected rut date by adding it with 22 days of rut cycle. 

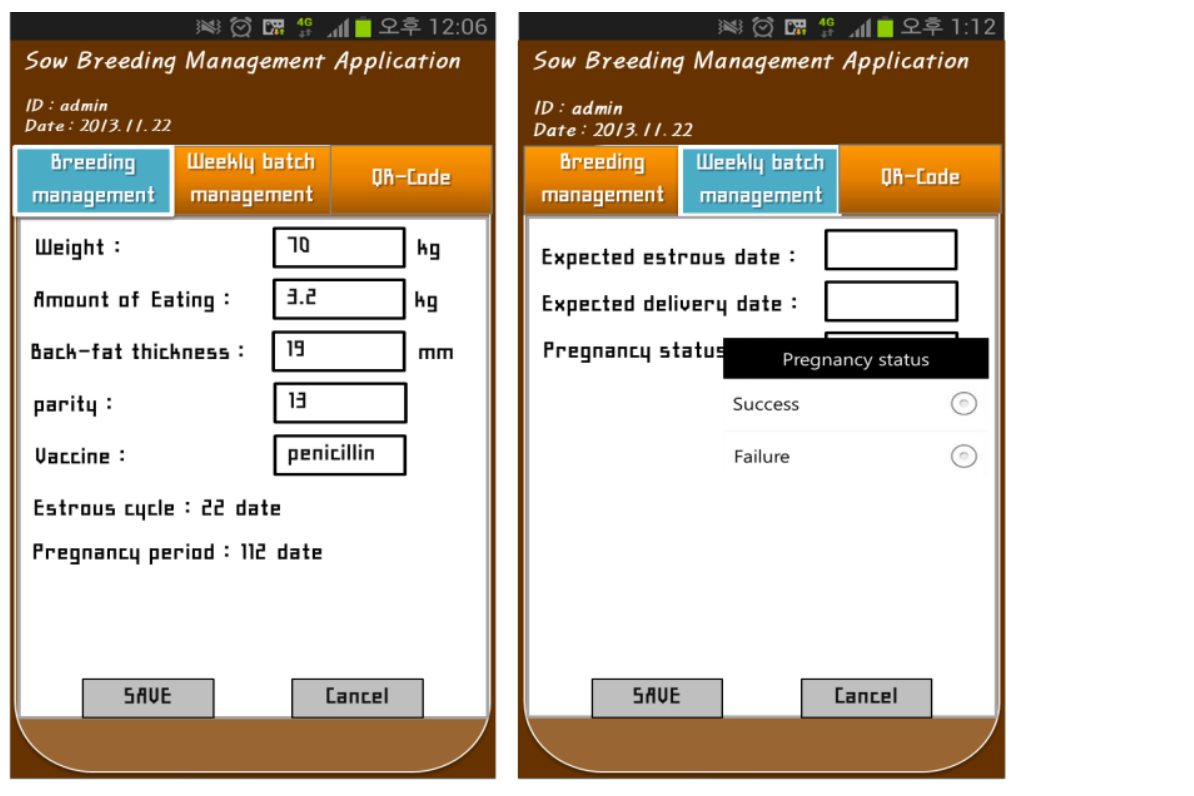

Figure 6. Implementation of the sow breedjngmanagement application system

\section{Conclusions}

The current circumstance is thatsystem ondomestic sow specification management is insufficient with severe individual differences, and the number of MSY remains to be low compared to that of advanced countries. To solve this, systematic specification management technology is needed. This paper designed a system for systematic specification management of sow and weekly management to increase the number of MSY of sow. Propose the system, artificial insemination during the optimal period of sow is possible As it has been designed based on QR code, it can solve the difficulty in using expensive RFID tag by livestock farms, as well as minimize the loss of bar code attached in sow room. In addition, it can provide convenience of user and is expected to improve the number of MSY of sow.

\section{Acknowledgements}

"This research was supported by the MSIP (Ministry of Science, ICT and Future Planning), Korea, und r the CITRC (Convergence Information Technology Research Center) support program (NIPA-2013-H0401-13-2008) supervised by the NIPA(National IT Industry Promotion Agenc)

"This research was financially supported by the Ministry of Education (MOE) and National Research Foundation of Korea (NRF) through the Human Resource Training Project for Regional Innovation (No.2011-0202 )."

\section{References}

[1] M. K. Kim, "According to the Korea-EU FTA avoid pork industry research services", The Association of Swine, Korea, (2010).

[2] K. H. Ryu, "Design and Implementation of Hog-Raising Population Management System using RFID", Incheon University, (2010).

[3] H. S. Heong and S. K. Heong and H. Yoe, "A Study on the Efficient Breeding Management System for Sow", Proceedings of KISM Spring Conference, (2013), pp. 230-233. 
[4] J. H. Hwang, "A Design of RFID/USN based Pig House Monitoring System for environmental conditions and bio-information”, Korea information and Communications Society, (2009), pp. 406-409.

[5] Y. D. Jang, "Comparison of Weekly and Batch Management System for Sows", Journal of livestock housing and environment, (2009), pp. 171-182.

[6] J. C. Park, "Efficient production of sows fertility management", Pig \& Pork Handon, (2012), pp. 258-261.

[7] J. H. Park, "A Research on Expansion of Library Service by Using QR Code", Libray and information Science, (2012), pp. 321-347.

\section{Authors}

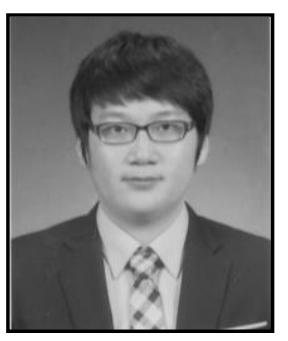

\section{Hansol Park}

$\mathrm{He}$ is a graduate school student of Sunchon National University. Hansol Park is also a researcher of u-agriculture IT Application Research Center and Agriculture IT Convergence Suppor Center at Sunchon National University too. Hansol Park's research focus is Wireless Sensor Networks, Android Application.

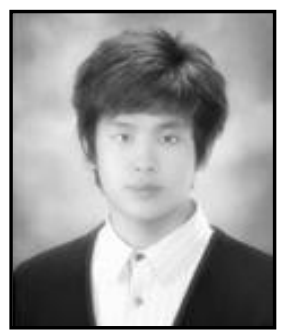

\section{Hoseok Jeong}

$\mathrm{He}$ is a graduate school sstudent of Sunchon National University. Hoseok Jeong (18)also a researcher of u-agriculture IT Application Research Center and Agriculture IT Convergence Support Center at Sunchon National University too. Hoseok Jeong's research focus is WirelessSensor Networks and Radio Frequency Identification.

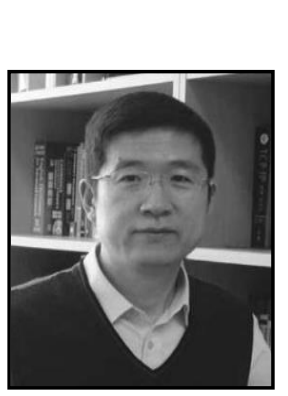

Hyun Yoe

$\mathrm{He}$ (1s a professor in the department of Information and communication at Sunchon National University, South Korea. He received his M.S and Ph.D degree in Electronic Engineering from Soongsil University in 1987 and 1992, respectively. He has been a research staff in KT research Institute in 1987-1993 and ETRI in 1995 in Korea. He also has been researched at Georgia Institute of Technology in 1997-1998 in U.S.A. He is a member of IEEE, KICS and KSII. Dr. Yoe's research focus is sensor networks and ubiquitous applications for agriculture. He has investigated issues in ubiquitous sensor networks and wireless networks. He has applied sensor networks to ubiquitous agriculture. He is in charge of uARC (ubiquitous Agriculture IT Application Research Center) and AITCSC(Agriculture IT Convergence Support Center) which is supported by MKE(Ministry of Knowledge Economy), Korea. 
International Journal of Multimedia and Ubiquitous Engineering Vol.9, No.4 (2014)

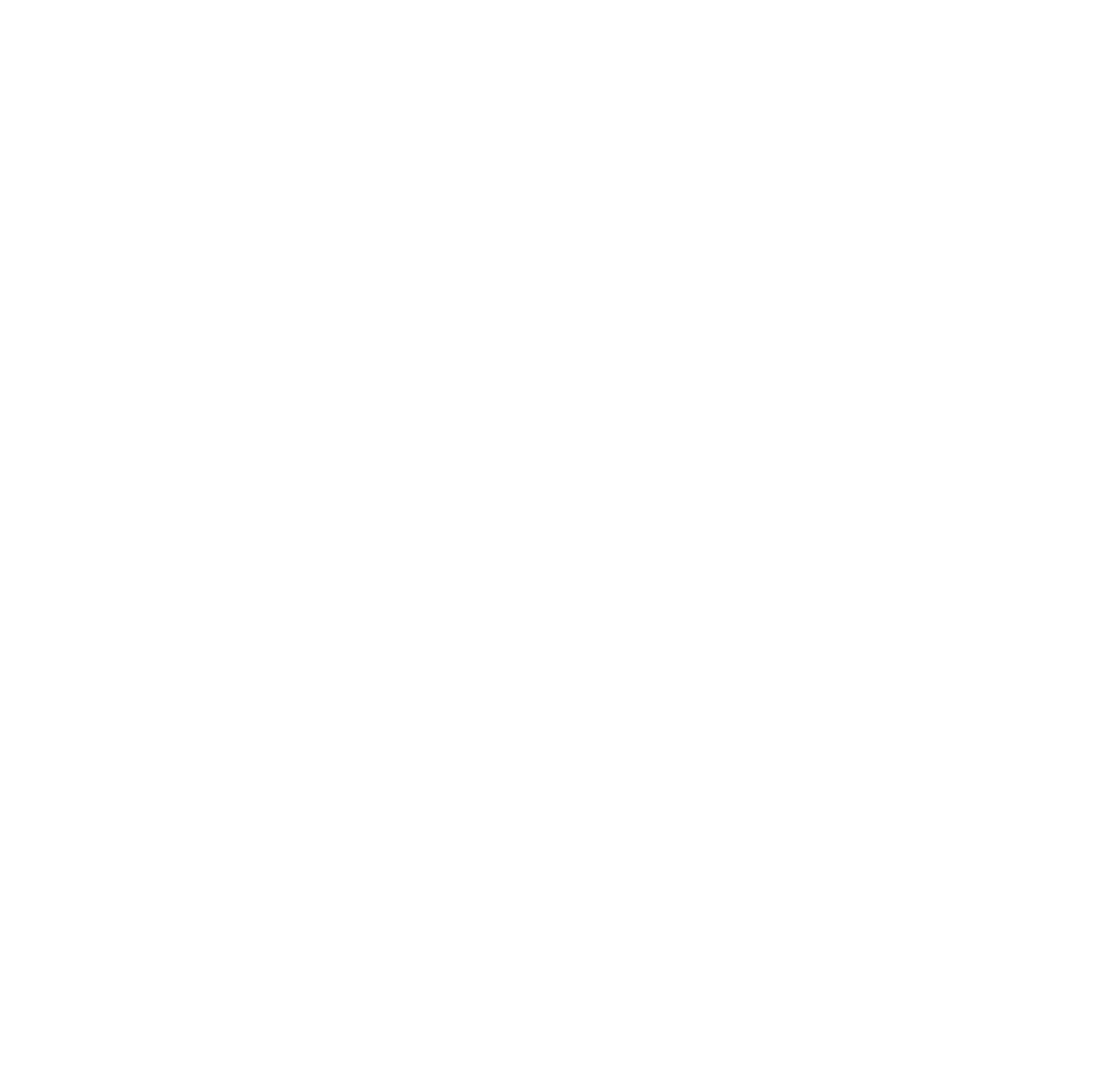

